\title{
Acknowledgement to the Reviewers
}

\section{Allergy ${ }_{\text {nd }}$ Immunology}

The Editors and the members of the editorial board would like to thank the following individuals for their expert assistance in acting as reviewers for Int Arch Allergy Immunol in the period of August 1, 2013 to December 31, 2014.
J. Buters, München

R.C. Aalberse, Amsterdam

K. Aasbjerg, Copenhagen

M. Abelius, Linköping

W. Aberer, Graz

K. Adel-Patient, Gif-sur-Yvette

I. Agache, Brasov

O. Akbari, Los Angeles, Calif.

M. Akdis, Davos

J.H. Akkerdaas, Amsterdam

C. Alessandri, Roma

I.C. Allen, Blacksburg, Va.

L.G. Arlian, Dayton, Ohio

M. Arock, Cachan

L. Arruda, Ribeirao Preto

H. Arshad, Southampton

R. Asero, Paderno Dugnano

M.B. Azad, Winnipeg, Man.

C. Bachert, Ghent

X.-F. Bai, Columbus, Ohio

B. Ballmer-Weber, Zürich

Fuad Baroody, Chicago, Ill.

Joan Bartra, Barcelona

H. Baurecht, Kiel

C. Bayerl, Wiesbaden

F. Bazzoni, Verona

A. Becker, Winnipeg, Man.

D. Becker, Mainz

M. Ben-Shoshan,

Montréal, Qué.

M. Bergmann, Genève

C. Berin, New York, N.Y.

L. Bianchetti, Basel

T. Biedermann, München

A. Bircher, Basel

J. Drew Bird, Dallas, Tex.

K. Blaser, Davos

K. Blümchen, Berlin

W.-H. Boehncke, Genève

M. Boita, Torino

L. Bouillet, Grenoble

L.-P. Boulet, Québec, Qué.

Arnaud Bourdin, Montpellier

F. Braido, Genova

E. Brandt, Cincinnati, Ohio

J. Brannan, Westmead, N.S.W.
B. Brashier, Pune

K. Brockow, München

P.J. Bryce, Chicago, Ill.

A.W. Burks, Chapel Hill, N.C.

R.K. Bush, Madison, Wis.

W.W. Busse, Madison, Wis.

J.T. Buters, München

A. Bygum, Odense

T. Caballero, Madrid

L. Calus, Ghent

R. Campana, Wien

D. Campbell, Sydney, N.S.W.

P. Campo, Málaga

M. Cancian, Padova

G. Walter Canonica, Genova

D. Carmona Cara, Belo Horizonte

V. Casolaro, Baronissi

M. Castells, Boston, Mass.

L. Cecchi, Firenze

A. Chaker, München

J. Chang, Zhunan

M. Chelminska, Gdansk

Y.-H. Chen, Taichung

F.T. Chew, Singapore

W. Chiang, Singapore

H.J. Chong Neto, Curitiba

A. Cianferoni,

Philadelphia, $\mathrm{Pa}$.

M. Cicardi, Milano

D.W. Cockcroft,

Saskatoon, Sask.

M. Comeau,

Thousand Oaks, Calif.

M.L. Conrad, Berlin

A. Cossarizza, Modena

L. Cox, Fort Lauderdale, Fla.

T. Craig, Hershey, Pa.

R. Crameri, Davos

M. Cugno, Milano

M. Curin, Wien

B. Dahlen, Huddinge

A. Daschner, Madrid

J.M. Davies, Brisbane, Qld.

R. De Bruyne, Ghent

D.J. DeBoer, Madison, Wis.
P. Demoly, Montpellier

L. Derycke, Gand

A. Diaz-Perales, Madrid

S. Dolff, Essen

S.C.C. Dreskin, Aurora, Colo.

C. Duez, Lille

B. Eberlein, München

M. Ebisawa, Kanagawa

M. Eibl, Wien

T. Eiwegger, Wien

L. Elfström, Uppsala

W. Emminger, Wien

E. Erwin, Columbus, Ohio

J. Esparza Gordillo, Berlin

S. Eyerich, München

P. Fallon, Dublin

Y. Fang, Des Moines, Iowa

N. Fens, Amsterdam

E. Fernandez-Caldas,

S. Sebastián de los Reyes

M. Ferrer, Pamplona

J.N. Fink, Milwaukee, Wis.

J. Fischer, Tübingen

M. Focke, Wien

L. Forbes, Houston, Tex.

P. Forsythe, Hamilton, Ont.

F. Frati, Milano

A. Freeman, Bethesda, Md.

Y. Fukutomi, Sagamihara

G. Gadermaier, Salzburg

E. Gadermaier, Wien

G.M. Gauvreau,

Hamilton, Ont.

R. Gehrig, Zürich

C. Georgalas, Amsterdam

R.G. van Wijk, Rotterdam

A. Geusau, Wien

P. Gevaert, Gand

B.F. Gibbs, Greenwich

G.J. Gleich,

Salt Lake City, Utah

M.J. Goikoetxea, Pamplona

D. Golden, Baltimore, Md.

E. González-Mancebo, Fuenlabrada

H.J. Gould, London
K. Grabmeier-Pfistershammer, Wien

C.E. Grattan, Colney, Norwich

B. Grimbacher, Freiburg

H. Grönlund, Stockholm

S. Guerra, Tucson, Ariz.

T.L. Hackett, Vancouver, B.C.

R. Handgretinger, Tübingen

S. Harsch, Stuttgart

K.T. HayGlass,

Winnipeg, Man.

G. Hedlin, Stockholm

E. Heffler, Torino

J. Heindrich, München

P. Hellings, Leuven

C. Hilger, Luxembourg

H. Hochwallner, Wien

H.J. Hoffmann, Aarhus

K. Hoffmann-Sommergruber, Wien

S.P. Hogan, Cincinnati, Ohio

M. Holmqvist, Uppsala

S.-J. Hong, Seoul

H.P. Horny, München

V. Hox, Antwerpen

A. Hsu, Newcastle

N. Inagaki, Gifu

H. Inoue, Kagoshima

K. Ito, Aichi

R. Jabeen, Indianapolis, Ind.

A. Jacquet, Bangkok

B. Jahn-Schmid, Wien

B. Jakiela, Krakow

D. Jankovic, Bethesda, Md.

L.J. Janssen, Hamilton, Ont.

U. Jappe, Borstel

J.C. Jensenius, Aarhus

D. Kabelitz, Kiel

L. Kalogjera, Zagreb

A.P. Kaplan, Charleston, S.C.

T. Katsunuma, Tokyo

Y. Katz, Zerifin

T. Keil, Berlin

M. Keller, Washington, D.C.

K.J. Kelly, Milwaukee, Wis.

D. Kesper, Marburg 
F. Kheradmand, Houston, Tex.

T. Kinaciyan, Wien

H. Kita, Rochester, Minn.

J. Kleine-Tebbe, Berlin

R. Klemans, Utrecht

E.F. Knol, Utrecht

Y. Kobayashi, Tokyo

M. Komine, Tochigi-ken

J. Koplin, Parkville, Vic.

Pe.T. Kovanen, Helsinki

S. Krauss-Etschmann, München

E. Krop, Utrecht

M. Kuitunen, Helsinki

M. Kulis, Chapel Hill, N.C.

R.K. Kumar, Sydney, N.S.W.

P. Lackner, Salzburg

H. Lapeere, Gand

S.T. Larsen, Copenhagen

A. Letran Camacho, Cádiz

F. Levi-Schaffer, Jerusalem

A. Lianbery,

Minneapolis, Minn.

J. Lidholm, Uppsala

V. Lombardi, Antony

A. Lopata, Townsville, Qld.

A. Lorentz, Stuttgart

O. Luengo, Barcelona

D.W. MacGlashan, Baltimore, Md.

B. Makay, Izmir

A. Mari, Latina

B.J. Marsland, Lausanne

J.G. Martin, Montréal, Qué.

S.F. Martin, Freiburg

P. Matricardi, Berlin

H. Matsuda, Fuchu

A. Matsuda, Tokyo

M. Maurer, Berlin

A. McIvor, Hamilton, Ont.

H. McSorley, Edinburgh

D. Mechtcheriakova, Wien

D. Metcalfe, Bethesda, Md.

M. Minkov, Wien

N. Mishra, Albuquerque, N.Mex.

R. Mösges, Köln

P. Moingeon, Antony
S. Molin, München

M. Moustaki, Athens

R.S. Mueller, München

L. Mueller, Basel

J. Mullol, Barcelona

M.A. Muraro, Padova

H. Murota, Suita

A. Nandy, Reinbek

H. Neighbour, Hamilton, Ont.

H.S. Nelson, Denver, Colo.

D. Neumann, Hannover

F. Nimmerjahn, Erlangen

I. Noss, Johnson City, Tenn.

A.H. Nowak-Wegrzyn,

New York, N.Y.

Y. Ohshima, Fukui

M. Ollert, München

L. O'Mahony, Davos

A. Orfao, Salamanca

J.N.G. Oude Elberink, Groningen

S. Pahr, Wien

N.G. Papadopoulos, Athens

H.-S. Park, Suwon

G. Passalacqua, Genova

S. Patil, Boston, Mass.

G. Pauli, Strasbourg

B. Pauwels, Gand

J.E. Pease, London

R.S. Peebles, Nashville, Tenn.

H.-H. Peter, Freiburg

W. Pfützner, Marburg

W.J. Pichler, Bern

W.F. Pickl, Wien

E. Pieckova, Bratislava

A. Planaguma,

Sant Feliu de Llobregat

A. Pomes, Charlottesville, Va.

P. Poncet, Paris

P. Porter, Houston, Tex.

W.S. Powell, Montréal, Qué.

D. Price, Aberdeen

C. Pucillo, Udine

U. Purath, Marburg

C. Rask, Hoersholm

I. Reese, München

K. Reich, Göttingen

K. Reich, Hamburg
A. Reshef, Tel Hashomer

N. Rezaei, Tehran

C. Rhyner, Davos

D.S. Robinson, London

G. Rolla, Torino

S. Ronchetti, Perugia

C. Rondon, Málaga

M. Rosewich, Frankfurt a.M.

F. Roth-Walter, Wien

K.H. Roux, Tallahassee, Fla.

F. Rueff, München

M. Ruiz Garcia, Madrid

S. Rutella, Roma

D. Ryan, Derby

V. Sabato, Antwerpen

J. Saloga, Mainz

A.J. Sandford, Vancouver, B.C.

V. Santarlasci, Firenze

A. Santos, London

G. Scadding, London

R. Schmitz, Berlin

L. Schwartz, Richmond, Va.

A.M. Scurlock,

Little Rock, Ark.

B.E. Sekerel, Ankara

M. Shamji, London

L.P. Shek, Singapore

S. Sicherer, New York, N.Y.

F. Siebenhaar, Berlin

T. Simon, Debrecen

S. Sjölander, Uppsala

H. Smits, Leiden

R. Sorkness, Madison, Wis.

M. Soyka, Zürich

M. Spangfort, Guangzhou

P. Staubach, Mainz

G. Stavroulakis, Athens

P. Steinberger, Wien

C. Steininger, Wien

C. Stellato, Baltimore, Md.

G. Stingl, Wien

G.J. Sturm, Graz

H. Su, Bethesda, Md.

I. Swoboda, Wien

Z. Szepfalusi, Wien

M. Tamari, Yokohama

S.J. Tebbutt, Vancouver, B.C.

Y. Teizo, Bethesda, Md.
A. ten Brinke, Leeuwarden

M.H. Ten Hacken, Groningen

D. Tey, Parkville, Vic.

J. Thalhamer, Salzburg

W. Thomas, West Perth, W.A.

S.F. Thomsen, Copenhagen

V. Thon, Brno

A. Thyagarajan, Richmond, Va.

M. Toda, Langen

S. Toppila-Salmi, Helsinki

E. Toubi, Haifa

M. Triggiani, Salerno

M. Tsai, Stanford, Calif.

F.M. Ubeira,

Santiago de Compostela

S. Ueki, Akita

H. van der Kleij, Leiden

R.J.J. van Neerven,

Wageningen

L.S. van Rijt, Amsterdam

M.T. Ventura, Bari

C. Vidal Pan, Santiago de

Compostela

S. Vieths, Langen

M. Villalba, Madrid

H. Vliagoftis, Edmonton, Alta.

T. Volz, Tübingen

S. Vrtala, Wien

D.V. Wallace,

Fort Lauderdale, Fla.

M. Wallner, Salzburg

S. Waserman, Hamilton, Ont.

B. Wedi, Hannover

R. Weiss, Salzburg

K. Weller, Berlin

M. Wickman, Stockholm

L. Willison, Tallahassee, Fla.

T. Witte, Hannover

S. Wöhrl, Wien

H.M. Wolf, Wien

J.S. Wolffsohn, Birmingham

G.W.K. Wong, Hong Kong

R.A. Wood, Baltimore, Md.

M. Worm, Berlin

M. Zemlin, Marburg

S. Zielen, Frankfurt

G. Zlabinger, Wien

L. Zuidmeer, Amsterdam 